\title{
Low-Loss 25.3km Few-Mode Ring-Core Fiber for Mode-Division Multiplexed Transmission
}

\author{
Yongmin Jung, Senior Member, IEEE, Qiongyue Kang, Hongyan Zhou, Rui Zhang, Su Chen, \\ Honghai Wang, Yucheng Yang, Xianqing Jin, Frank P. Payne, Shaif-Ul Alam, and \\ David J. Richardson, Fellow, IEEE,
}

\begin{abstract}
We report the design, fabrication and characterization of a few-mode ring-core fiber supporting 4 mode groups (i.e. 7 spatial modes including spatial degeneracies). By carefully designing the ring-core parameters, the fiber can support only single-radial-order modes, which enables weak intermodal coupling between higher-order modes and has potential to reduce the complexity of mode-division multiplexed digital signal processing. The low loss $(\sim 0.3 \mathrm{~dB} / \mathrm{km})$ and long length $(25.3 \mathrm{~km})$ $\mathrm{RCF}$ is successfully fabricated and they are both records for a ring-core fiber.
\end{abstract}

Index Terms - Few-mode fibers, ring-core fiber, mode-division multiplexing, space-division multiplexing.

\section{INTRODUCTION}

$\mathrm{M}$ ode division multiplexing (MDM) has attracted considerable attention in the fiber-optic community as a promising approach to increase the per-fiber capacity by employing multiple distinguishable spatial information channels within the same multimode core [1-4]. Several different types of few-mode fiber (FMF) [5-9] have been proposed and investigated to date. In the vast majority of FMF transmission systems, multiple-input, multiple-output (MIMO) digital signal processing (DSP) is an essential requirement in order to compensate for the linear cross-talk between optical modes that ordinarily occurs due to mode coupling. As the number of modes (information channels) increases, the complexity of the MIMO processing required increases rapidly for conventional step-index or graded-index FMFs $[10,11]$. However, if mode coupling can be reduced the use of MIMO processing might be considerably simplified (or possibly even avoided), thereby increasing the viability and scalability of the MDM approach.

Manuscript received October 23, 2016. This work was supported in part by the UK EPSRC grant EP/J008591/1 (COMIMO) and the Hubei, China 2015 CFB705 funding. Data contained in this paper is openly available from the University of Southampton repository at: http://dx.doi.org/10.5258/SOTON/XXXXXX.

Y. Jung, Q. Kang, S. Alam, D. J. Richardson are with the Optoelectronics Research Centre, University of Southampton, Southampton, SO17 1BJ, U.K. (e-mail: ymj@orc.soton.ac.uk, qk1g11@orc.soton.ac.uk, sua@orc.soton.ac.uk, djr@orc.soton.ac.uk).
In this aspect, few-mode ring-core fibers (FM-RCFs) that support single-radial-order modes (i.e. $\mathrm{LP}_{1 \mathrm{~m}}$ modes where $m$ is an integer) have been reported both theoretically [12-14] and experimentally [15-21] to show great potential for improving the transmission capacity of MDM system with low DSP complexity. In FM-RCFs, the effective index difference between adjacent neighbouring azimuthal modes significantly increases with increasing azimuthal mode number, which can result in relatively weak mode coupling between higher-order azimuthal modes. Therefore, the DSP complexity can be reduced by using MIMO processing only to recover signals carried on those lower-order azimuthal modes which experience strong mode coupling and/or between modes within the same mode group [15-17]. In addition, ring-core fiber amplifiers can, in theory, provide nearly identical gain for all guided signal modes owing to the fact that similar overlap factors can be achieved between the erbium doped core and all the signal spatial modes [22-24]. RCF amplifiers are thus very attractive as MDM amplifiers in terms of having low mode dependent gain. However, despite the aforementioned merits of FM-RCFs the development of long lengths of suitably low loss RCF has proved a challenge. For example, the 7 mode-group RCF reported early in 2015 suffered from a substantial fiber attenuation of a few hundred $\mathrm{dB} / \mathrm{km}$ [15] and even the most recent results describing the development of a 5 mode-group $\mathrm{RCF}$ reported a fiber attenuation of a few $\mathrm{dB} / \mathrm{km}$ [16]. It is therefore clear that fiber loss must be driven down in order to make FM-RCFs a feasible approach for high-capacity longdistance MDM transmission.

In this work, we have designed and successfully fabricated a $25.3 \mathrm{~km}$ length of low loss FM-RCF supporting 4 mode groups (i.e. 7 spatial modes including all spatial degeneracies). The fiber attenuation for all guided modes was around $0.3 \mathrm{~dB} / \mathrm{km}$, which is the lowest fiber loss so far reported in the FM-RCF family. To fully quantify the modal properties of the fiber, the modal intensity profiles, mode dependent loss and multimode temporal impulse response were also investigated.

X. Jin and F. Payne are with the Department of Engineering Science, University of Oxford, OX1 3PJ, U.K. (e-mail: xqjin@ustc.edu.cn, frank.payne@lincoln.ox.ac.uk).

H. Zhou, R. Zhang, S. Chen, H. Wang and Y. Yang are with the State Key Laboratory of Optical Fiber and Cable Manufacture Technology, Yangtze Optical Fiber and Cable Joint Stock Limited Company, Wuhan, China (e-mail: zhouhongyan@yofc.com, zhangrui@yofc.com, chensu@yofc.com, wanghonghai@yofc.com, yangyucheng@yofc.com).

Color versions of one or more of the figures in this paper are available online at http://ieeexplore.ieee.org.

Digital Object Identifier XX.XXXX/JLT.2016.XXXXXXX 

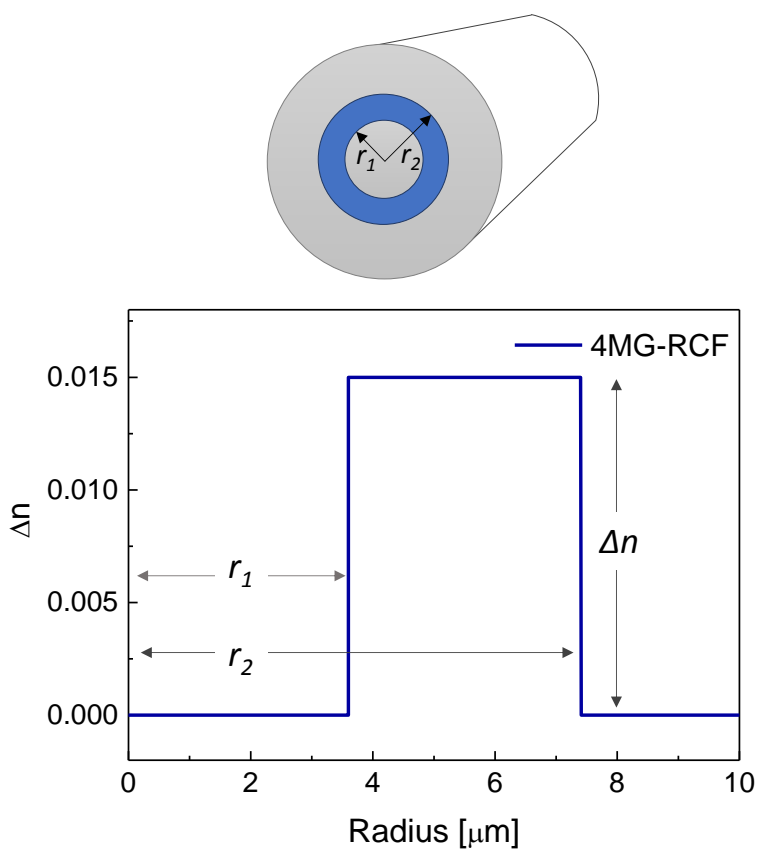

(a)
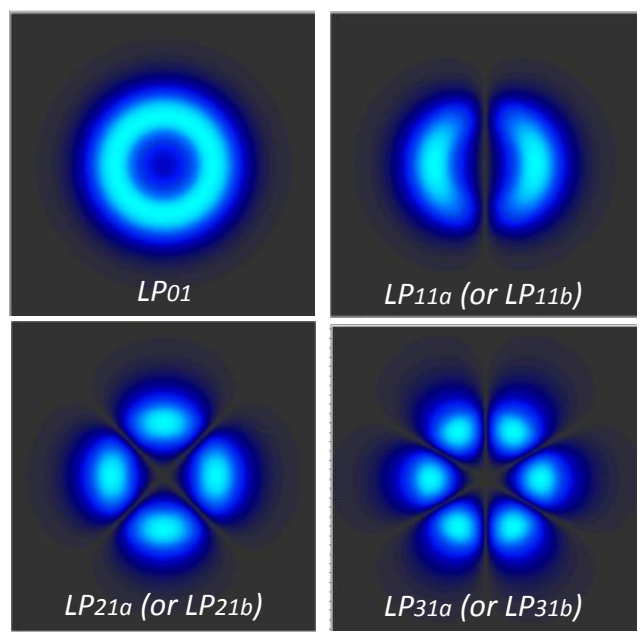

(b)

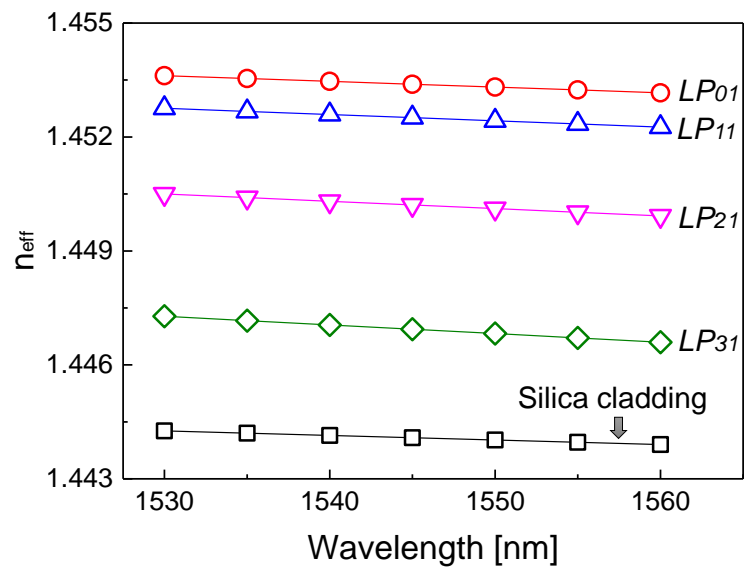

(c)

Fig. 1. (a) The refractive index profile of the RCF design that supports 4 mode groups (7 spatial modes), (b) the intensity distribution of the guided modes of the RCF at $\lambda=1550 \mathrm{~nm}$. (c) Effective refractive indices of the guided modes as a function of wavelength.

\section{FIBER DESIGN AND FABRICATION}

A step-index RCF is defined by two structural design parameters, i.e. $r_{1}$ and $r_{2}$, which define the two boundaries of the ring core, as well as the index difference $\Delta \mathrm{n}$, as shown in Fig. 1(a). In this paper, we propose a 4 mode-group RCF (4MG$\mathrm{RCF}$ ) design, whose fiber refractive index profile (FRIP) is shown in Fig. 1(a) with parameters $r_{1}=3.8 \mu \mathrm{m}, \mathrm{r}_{2}=7.3 \mu \mathrm{m}$ and $\Delta \mathrm{n}=0.015$. The key design objective for this $4 \mathrm{MG}-\mathrm{RCF}$ was to ensure strong guidance for the $\mathrm{LP}_{01}, \mathrm{LP}_{11}, \mathrm{LP}_{21}$, and $\mathrm{LP}_{31}$ mode groups in the $\mathrm{C}$-band, whilst the next higher order mode, i.e. $\mathrm{LP}_{41}$, is completely cut-off at $\lambda=1500 \mathrm{~nm}$. Generally, fiber attenuation is strongly related to the macro-bending and/or micro-bending loss of the guided modes and a relatively large effective index difference $\left(\Delta \mathrm{n}_{\text {eff }}\right)$ between the guided modes and cladding modes is essential to reduce the intrinsic fiber loss by suppressing mode coupling from the guided $\mathrm{LP}_{31}$ mode to leaky cladding modes. As shown in the modal intensity distribution in Fig. 1(b), the four guided mode groups are well confined in the ring core area and the simulated power fractions in the core for the $\mathrm{LP}_{01}, \mathrm{LP}_{11}, \mathrm{LP}_{21}$ and $\mathrm{LP}_{31}$ mode groups at $1550 \mathrm{~nm}$ are $91 \%, 92 \%, 91 \%$ and $88 \%$, respectively. Figure 1 (c) shows the simulated modal effective indices of the 4MG-RCF in the wavelength range $1530-1560 \mathrm{~nm}$. The normalized propagation

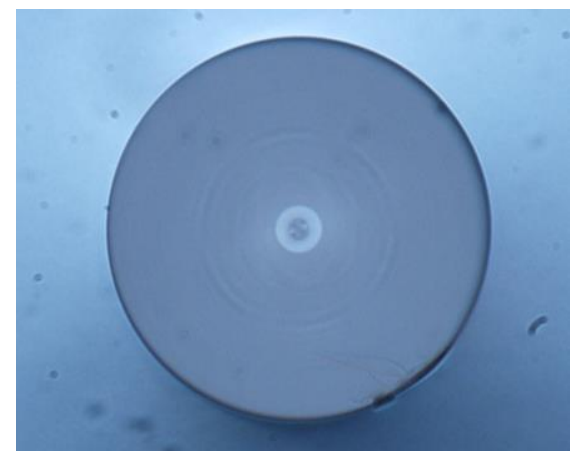

(a)

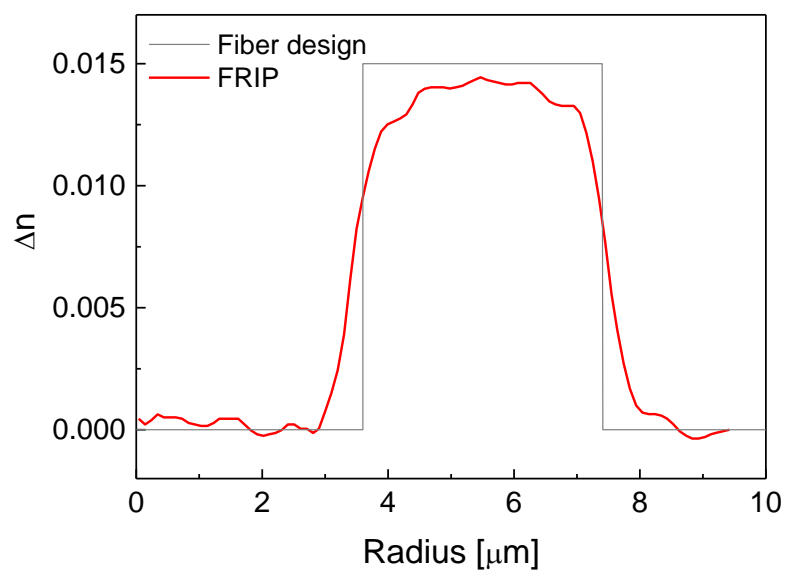

(b)

Fig. 2. (a) The fiber cross-section and (b) measured FRIP (in red line) of the fabricated $4 \mathrm{MG}-\mathrm{RCF}$. 
constant $b=\frac{n_{e f f^{-} n_{2}^{2}}}{n_{1}^{2}-n_{2}^{2}}$ of the $\mathrm{LP}_{31}$ mode group at 1550nm in the designed $4 \mathrm{MG}-\mathrm{RCF}$ is 0.23 , which also provides for good guidance of the modes. The effective index difference between the $\mathrm{LP}_{01}$ and $\mathrm{LP}_{11}$ modes is about $8 \times 10^{-4}$, while the differences between other higher-order mode groups is more than $2 \times 10^{-3}$. This large effective index difference between adjacent HOMs can assure weak mode coupling between the HOMs, although relatively strong mode coupling between the $\mathrm{LP}_{01}$ and $\mathrm{LP}_{11}$ mode group is to be expected.

Using a conventional plasma chemical vapour deposition (PCVD) process, we have successfully fabricated the $4 \mathrm{MG}-$ RCF. Figure 2(a) shows an optical microscope image of the cross section of the fabricated RCF. The fiber core is clearly observed as a bright annular ring with a dark spot at its center because white light from a halogen lamp can be transmitted through the core of the fiber. The fiber refractive index profile of the fabricated RCF (red line in Fig. 2(b)) is reasonably well matched to the fiber design (gray line). The fiber has an inner core radius $\left(r_{1}\right)$ of $3.4 \mu \mathrm{m}$, outer core radius $\left(r_{2}\right)$ of $7.5 \mu \mathrm{m}$, $\Delta \mathrm{n}=0.0135$ and an outer cladding dimeter of $125 \mu \mathrm{m}$.

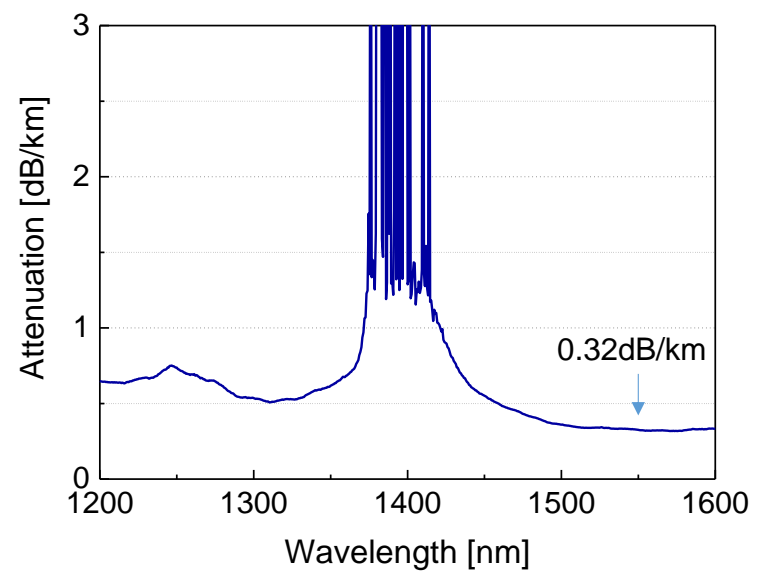

(a)

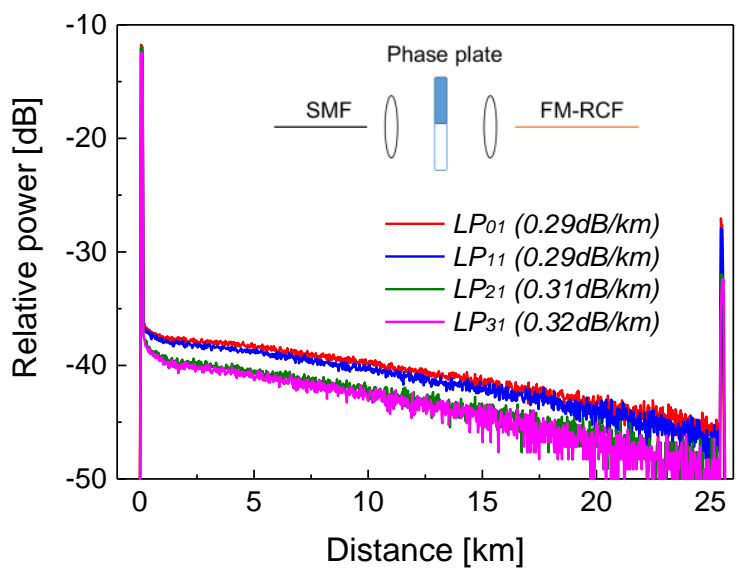

(b)

Fig. 3. (a) Averaged fiber loss (using cut-back method) and (b) mode dependent fiber loss (using mode selective OTDR) of the RCF.

\section{MODAL CHARACTERIZATION OF THE FM-RCF}

\section{A. Fiber attenuation}

First of all, the mode-averaged fiber attenuation was measured by a cut-back method using a white light source and an optical spectrum analyzer. Conventional $50 \mu \mathrm{m}$ step-index multimode fiber pigtails were spliced at both input/output ends of the RCF to provide over-filled light launching conditions into the RCF, which provides us with an averaged fiber loss over all spatial modes. As shown in Fig. 3(a), the fabricated RCF exhibits an averaged fiber attenuation of $0.32 \mathrm{~dB} / \mathrm{km}$ at $1550 \mathrm{~nm}$ (i.e. 8.2 $\mathrm{dB}$ span loss over $25.3 \mathrm{~km}$ ), which is the lowest loss value ever reported for a ring-core fiber. Water absorption peaks appeared at $\sim 1240 \mathrm{~nm}$ and $\sim 1380 \mathrm{~nm}$ due to the presence of $\mathrm{OH}$ ion impurities but these could easily be reduced by adopting a "dry fiber" fabrication process in the future.

To examine the modal dependency of the fiber attenuation an optical time domain reflectometer (OTDR) was used in conjunction with a mode selective excitation scheme based on phase plates. As shown in the inset of Fig. 3(b), a suitable phase plate is used to selectively launch a specific mode of the RCF and the reflected Rayleigh back-scattered light was analyzed using the same phase plate, which enabled us detect backscattered light from the same spatial mode. Therefore, using this mode-selective excitation/detection scheme, we can analyze the mode dependent loss of the fiber using a standard single-mode fiber OTDR. Figure 3(b) shows the OTDR traces

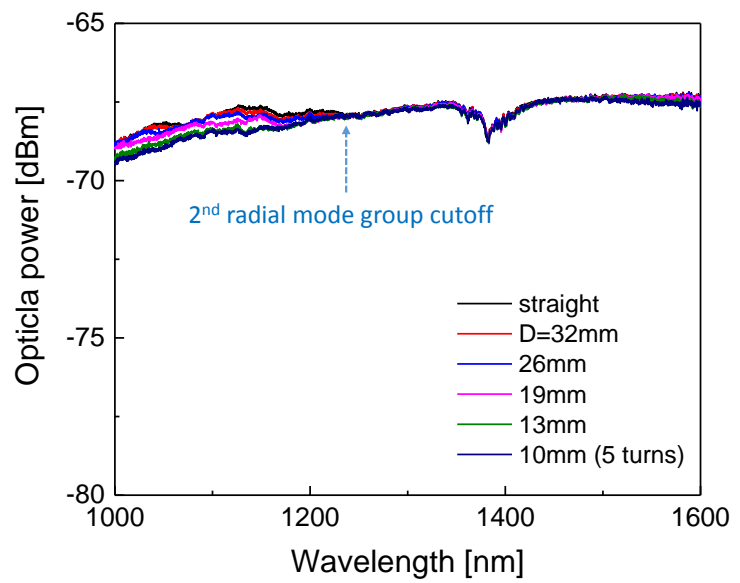

(a)

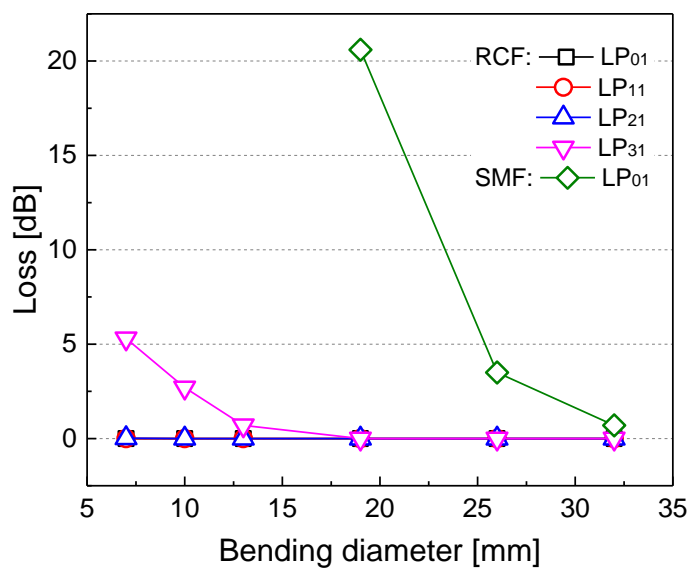

(b)

Fig. 4. (a) Average bend loss spectra and (b) mode dependent bend loss of the RCF (measured using phase plate based mode excitation). 
for four guided mode groups of the fiber (i.e. $\mathrm{LP}_{01}, \mathrm{LP}_{11}, \mathrm{LP}_{21}$, and $\mathrm{LP}_{31}$ ) which are excited selectively one-by-one using the corresponding phase plates. All spatial modes show similar propagation losses $(\sim 0.3 \mathrm{~dB} / \mathrm{km})$ and negligible polarization dependent losses. The OTDR measurements coincide with and are quite well matched to measurements made previously using the cutback method. A slightly higher loss was observed for higher-order modes (HOMs) but the differences are very modest.

\section{B. Macro-bending loss}

The macro-bending properties of the fabricated RCF were examined by winding 5-turns of the fiber onto mandrels of different diameter. First, all spatial modes of the RCF were excited by splicing a step-index $50 \mu \mathrm{m}$-core MMFs at both fiber ends and transmission spectra were recorded with a white light source. As shown in Fig. 4(a), the transmission spectrum of fiber at small bending diameters was almost the same as for straight fiber in the range of $1500-1600 \mathrm{~nm}$ and the average bend loss at these wavelengths remains relatively low. Interestingly, a discrete transmission power drop was observed in Fig. 4(a) at wavelengths around $1240 \mathrm{~nm}$ under fiber bending and we believe that this is related to the cut-off wavelength of the second radial mode group (e.g. $\left.\mathrm{LP}_{02}, \mathrm{LP}_{12}, \mathrm{LP}_{22} \ldots\right)$. Similarly to the $\mathrm{LP}_{11}$ mode cut-off in a single mode fiber design, the second radial mode group cut-off should be properly designed to provide a suitable balance between guidance strength (or core confinement) and ensuring robust single radial mode guidance conditions. Compared to our previously fabricated RCF reported in Ref. [15], a smaller ring diameter and a thicker ringcore were chosen for the current RCF. The measured second radial mode cut-off was shifted considerably (from $870 \mathrm{~nm}$ to $1240 \mathrm{~nm}$ ) and the fiber attenuation was greatly improved. The detailed fiber design considerations are described in ref. [12].

Secondly, we also tested the mode dependent bending loss at $1550 \mathrm{~nm}$ using a phase-plate based mode excitation approach. As shown in Fig. 4(b), all spatial modes of the RCF show high bending robustness and the bend loss of the $\mathrm{LP}_{31}$ mode, the highest order mode of the fiber, is much smaller than that of conventional SMF. In our proposed fiber design (Fig. 1(c)), the large effective index difference between the guided core modes and the cladding modes enables low macro-bend loss sensitivity.

\section{Multimode impulse response}

We performed time-of-flight (ToF) measurements [9] on the 4MG-RCF under selective mode excitation to further characterize the multimode fiber impulse response, in particular the differential group delay (DGD) of the different spatial modes. The DGD over the full $25.3 \mathrm{~km}$ length of 4MG-RCF was too large to be unambiguously measured using the available equipment and hence a $1 \mathrm{~km}$ length of fiber was taken to accurately measure the DGDs of the fiber. The traces in Fig. 5(a) show the four main distinct and discernible mode peaks at their relative DGD locations $\left(3.9 \mathrm{ps} / \mathrm{m}\right.$ for $\mathrm{LP}_{11}, 11.0 \mathrm{ps} / \mathrm{m}$ for $\mathrm{LP}_{21}$, and $18.2 \mathrm{ps} / \mathrm{m}$ for $\mathrm{LP}_{31}$ ), which agrees very well with our simulations. To find out the modal identity of the individual peaks in the ToF measurement the output beam intensity from the RCF was examined using a charge coupled device (CCD) to identify the dominant spatial guided mode under the selective mode excitation. The $\mathrm{LP}_{01}, \mathrm{LP}_{11}, \mathrm{LP}_{21}$ and $\mathrm{LP}_{31}$ spatial modes are clearly identified after $1 \mathrm{~km}$ of fiber as shown in Fig. 5(b). The near field distribution was measured using a microscope objective lens $(\times 50)$ to magnify the spatial modes onto a CCD camera placed at the focus. By moving the fiber slightly away from the focal point (i.e. by defocusing), the far field distribution can also be measured. As expected the measured modal intensities showed an annular ring shaped profile in the near field (e.g. a ring-shaped $\mathrm{LP}_{01}$ mode) but these evolved to the more usual fiber transverse mode profiles in the far field (e.g. a Gaussian-shaped $\mathrm{LP}_{01}$ mode). More importantly, we
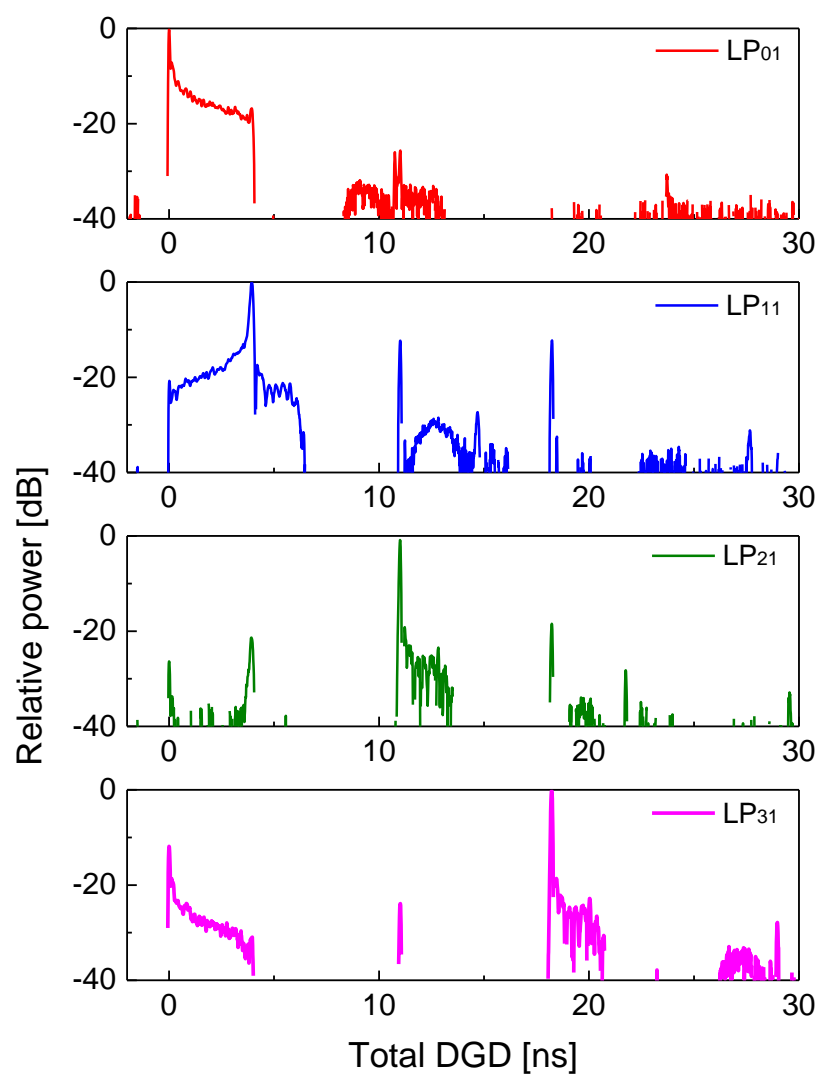

(a)

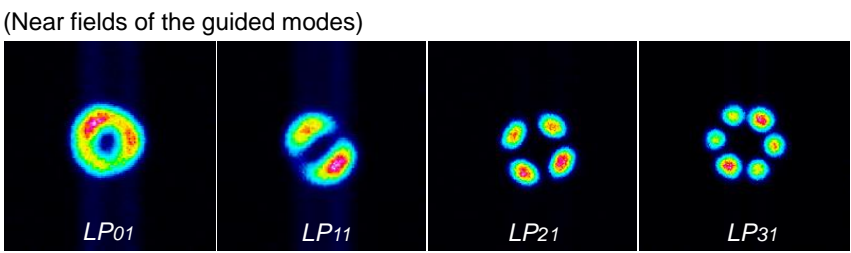

(Far fields of the guided modes)

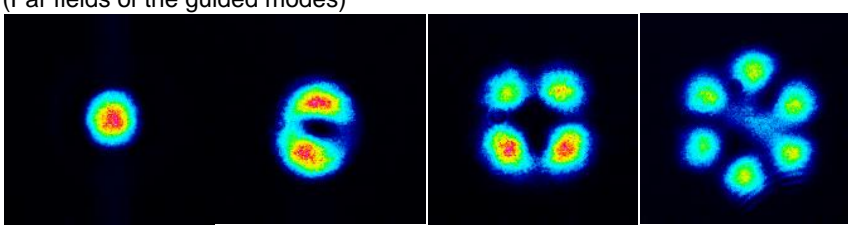

(b)

Fig. 5. (a) Time-of-flight measurement of the 4MG-RCF under selective mode excitation and (b) the beam intensity (near and far fields) of the guided modes.

have observed relatively strong mode coupling between the $\mathrm{LP}_{01}$ and $\mathrm{LP}_{11}$ mode groups in Fig. 5(a), which is evidenced by a flat plateau between these two mode groups for delays between 0 and $3.9 \mathrm{~ns}$. Under $\mathrm{LP}_{01}$ mode excitation, for example, a discrete peak was observed at $0 \mathrm{~ns}$ but a smooth sloped plateau was noticed towards the $\mathrm{LP}_{01}$ peak due to the strong distributed mode coupling occurring along the entire length of fiber. Under 
$\mathrm{LP}_{11}$ mode excitation, however, this plateau is now sloped toward the $\mathrm{LP}_{11}$ peak but with an almost identical magnitude of gradient due to the symmetric mode coupling. About $50 \%$ of the optical power resides in the distributed plateau after $1 \mathrm{~km}$ fiber propagation corresponding to a modal coupling efficiency of $0.5 \mathrm{~km}^{-1}$. However, there is no noticeable plateau between other higher-order mode groups in the RCF. This interesting mode coupling feature can be easily understood from the modal effective index calculation of the RCF in Fig. 1(c). The $\Delta \mathrm{n}_{\text {eff }}$ between the $\mathrm{LP}_{01}$ and $\mathrm{LP}_{11}$ group is relatively small and this results in strong mode coupling. However, the large $\Delta \mathrm{n}_{\text {eff }}$

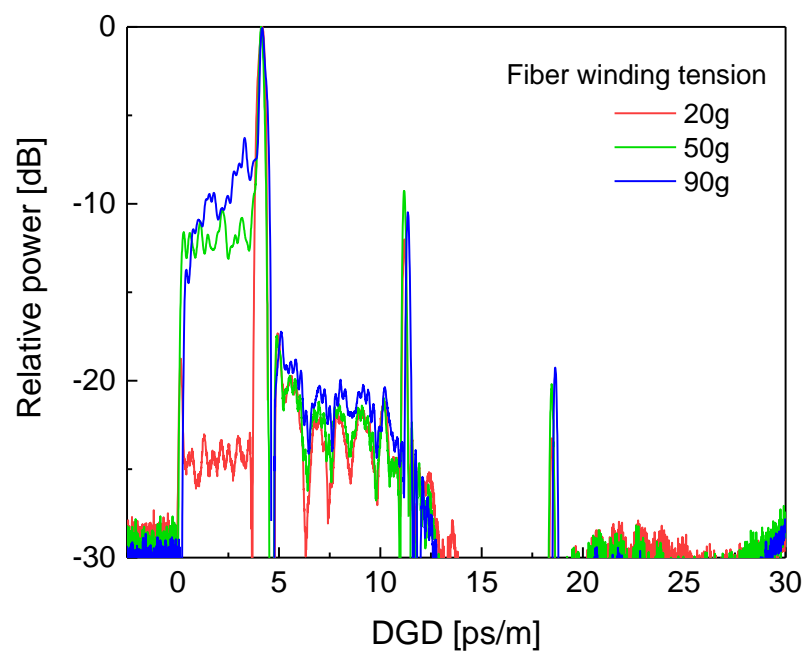

(a)

Fig. 6. Time-of-flight measurement of the 4MG-RCF under various fiber rewind tensions. The strength of the mode coupling between lower-order modes was affected by the micro-bending induced fiber winding tension.

between other HOMs in RCFs prevents distributed mode coupling between neighboring modes.

We also tested the ToF traces using a $25.3 \mathrm{~km}$ length of 4MG$\mathrm{RCF}$ and there was no discernible distributed mode coupling between HOMs (less than $-30 \mathrm{~dB}$ ). We plan to carry out an MDM transmission experiment using this $25.3 \mathrm{~km} 4 \mathrm{MG}-\mathrm{RCF}$ to validate the transmission performance and the required level of MIMO DSP complexity reduction in the near future.

\section{Micro-bending property}

Finally, the micro-bending sensitivity of the RCF was examined by rewinding the $300 \mathrm{~m}$ length of fiber onto a bobbin (diameter $=15 \mathrm{~cm}$ ) under various amounts of tension. No significant micro-bending induced optical loss was observed on the fabricated RCF however a significant increase in intermodal crosstalk was noticed in the time-of-flight measurement as shown in Fig. 6. The strength of the distributed mode coupling between the $\mathrm{LP}_{01}$ and $\mathrm{LP}_{11}$ mode (i.e. DGD between 0 and 3.9 $\mathrm{ps} / \mathrm{m}$ ) gradually increased with increasing winding tension from $20 \mathrm{~g}$ to $50 \mathrm{~g}$ but with the strength of coupled power sloping more towards the $\mathrm{LP}_{01}$ peak at $90 \mathrm{~g}$ of tension. It appears that the fiber winding tension is an important factor in determining the intermodal crosstalk in the fiber and that the intermodal coupling coefficient can be changed from a weakly coupled regime to a strongly coupled regime by control of the fiber winding tension.

\section{CONCLUSIONS}

We have designed and fabricated a low-loss few-mode ringcore fiber supporting 4 mode groups. All spatial modes show a similar fiber attenuation of $\sim 0.3 \mathrm{~dB} / \mathrm{km}$, which is the lowest loss value ever reported for a ring-core fiber. Due to the large effective index separation between the neighboring higherorder modes, the distributed intermodal coupling can be minimized and this should be very beneficial in terms of reducing the MIMO DSP complexity required for MDM transmission. In addition, the macro- and micro-bending properties of the ring core fiber was further investigated in terms of mode dependent loss and intermodal coupling.

\section{REFERENCES}

[1] D. J. Richardson, J. M. Fini, and L. E. Nelson, "Space-division multiplexing in optical fibres," Nat. Photonics, vol. 7, pp. 354-362, 2013.

[2] D. J. Richardson, "New optical fibres for high-capacity optical communications Subject Areas :," Philos. Trans. R. Soc. A, vol. 374, p. 20140441, 2016

[3] G. Li, N. Bai, N. Zhao, and C. Xia, "Space-division multiplexing: the next frontier in optical communication," Adv. Opt. Photonics, vol. 6, no. 4, pp. 413-487, Dec. 2014.

[4] P. J. Winzer, "Making spatial multiplexing a reality," Nat. Photonics, vol. 8, no. 5, pp. 345-348, Apr. 2014.

[5] Pierre Sillard, Marianne Bigot-Astruc, and Denis Molin, "Few-Mode Fibers for Mode-Division-Multiplexed Systems," J. Lightwave Technol. 32, 2824-2829 (2014).

[6] L. Grüner-Nielsen, Y. Sun, J. W. Nicholson, D. Jakobsen, K. G. Jespersen, R. Lingle, Jr., and B. Pálsdóttir, "Few Mode Transmission Fiber With Low DGD, Low Mode Coupling, and Low Loss," J. Lightwave Technol. 30, 3693-3698 (2012).

[7] S. Jain, Y. Jung, T. C. May-Smith, S. U. Alam, J. K. Sahu, and D. J. Richardson, "Few-mode multi-element fiber amplifier for mode division multiplexing," Opt. Express 22, 29031-29036 (2014).

[8] S. Ramachandran and P. Kristensen, "Optical vortices in fiber," Nanophotonics 2(5-6), 455-474 (2013).

[9] V.A.J.M. Sleiffer, Y. Jung, N. K. Baddela, J. Surof, M. Kuschnerov, V. Veljanovski, J. R. Hayes, N. V. Wheeler, E. R. N. Fokoua, J. P. Wooler, D. R. Gray, N. H. L. Wong, F. R. Parmigiani, S. Alam, M. N. Petrovich, F. Poletti, D. J. Richardson, and H. de Waardt, "High capacity modedivision multiplexed optical transmission in a novel 37-cell hollow-core photonic bandgap fiber," J. Lightwave Technol. 32, 854-863 (2014).

[10] N. K. Fontaine, R. Ryf, H. Chen, A. V. Benitez, B. Guan, R. Scott, B. Ercan, S. J. B. Yoo, L. E. Grüner-Nielsen, Y. Sun, R. Lingle, E. AntonioLopez, and R. Amezcua-Correa, "30×30 MIMO Transmission over 15 Spatial Modes," in Optical Fiber Communication Conference Post Deadline Papers, OSA Technical Digest (online) (Optical Society of America, 2015), paper Th5C.1.

[11] R. Ryf, N. K. Fontaine, H. Chen, B. Guan, S. Randel, N. Sauer, S. J. B. Yoo, A. Koonen, R. Delbue, P. Pupalaikis, A. Sureka, R. Shubochkin, Y. Sun, and R. Lingle, "23 Tbit/s Transmission over 17-km Conventional 50 um Graded-Index Multimode Fiber," in Optical Fiber Communication Conference: Postdeadline Papers, (Optical Society of America, 2014), paper Th5B.1.

[12] Xianqing Jin, Ariel Gomez, Kai Shi, Benn C. Thomsen, Feng Feng, George S. D. Gordon, Timothy D. Wilkinson, Yongmin Jung, Qiongyue Kang, Pranabesh Barua, Jayanta Sahu, Shaif-ul Alam, David J. Richardson, Dominic C. O'Brien, and Frank P. Payne, "Mode Coupling Effects in Ring-Core Fibers for Space-Division Multiplexing Systems," J. Lightwave Technol. 34, 3365-3372 (2016).

[13] M. Kasahara, K. Saitoh, T. Sakamoto, Nobutomo Hanzawa, Takashi Matsui, Kyozo Tsujikawa, and Fumihiko Yamamoto, "Design of ThreeSpatial-Mode Ring-Core Fiber," J. Lightwave Technol. 32, 1337-1343 
(2014)

[14] C. Brunet, B. Ung, P. Bélanger, Y. Messaddeq, S. LaRochelle, and L. Rusch, "Vector Mode Analysis of Ring-Core Fibers: Design Tools for Spatial Division Multiplexing," J. Lightwave Technol. 32, 4046-4057 (2014)

[15] F. Feng, G. S. Gordon, X. Q. Jin, D. C. O'Brien, F. P. Payne, Y. Jung, Q. Kang, J. K. Sahu, S. U. Alam, D. J. Richardson, and T. D. Wilkinson, "Experimental Characterization of a Graded-Index Ring-Core Fiber Supporting 7 LP Mode Groups," in Optical Fiber Communication Conference, OSA Technical Digest (online) (Optical Society of America, 2015), paper Tu2D.3.

[16] F. Feng, X. Guo, G. S. Gordon, X. Jin, F. Payne, Y. Jung, Q. Kang, S. Alam, P. Barua, J. Sahu, D. J. Richardson, I. H. White, and T. D. Wilkinson, "All-optical Mode-Group Division Multiplexing Over a Graded-Index Ring-Core Fiber with Single Radial Mode," in Optical Fiber Communication Conference, OSA Technical Digest (online) (Optical Society of America, 2016), paper W3D.5.

[17] K. Shi, A. Gomez, X. Jin, Y. Jung, C. Quintana, D. O'Brien, F. Payne, P. Barua, J. Sahu, Q. Kang, S. Alam, D. J. Richardson, and B. C. Thomsen, "Simplified Impulse Response Characterization for Mode Division Multiplexed Systems," in Optical Fiber Communication Conference, OSA Technical Digest (online) (Optical Society of America, 2016), paper W4F.3.

[18] T. Mori, T. Sakamoto, M. Wada, A. Urushibara, T. Yamamoto, and K. Nakajima, "Strongly-Coupled Two-LP-mode Ring-Core Fiber with Optimized Index Profile Considering S-bend Model," in Optical Fiber Communication Conference, OSA Technical Digest (online) (Optical Society of America, 2016), paper W1F.6.

[19] N.K. Fontaine, R. Ryf, M. Hirano, T. Sasaki, "Experimental Investigation of Crosstalk Accumulation in a Ring-Core Fiber", IEEE Summer Topicals, (USA, 2013), Paper TuC4.2.

[20] R. M. Nejad, K. Allahverdyan, P. Vaity, S. Amiralizadeh, C. Brunet, Y. Messaddeq, S. LaRochelle, and L. A. Rusch, "Mode Division Multiplexing Using Orbital Angular Momentum Modes Over 1.4-km Ring Core Fiber," J. Lightwave Technol. 34, 4252-4258 (2016).

[21] B. Ung, P. Vaity, L. Wang, Y. Messaddeq, L. A. Rusch, and S. LaRochelle, "Few-mode fiber with inverse-parabolic graded-index profile for transmission of OAM-carrying modes," Opt. Express 22, 18044-18055 (2014).

[22] Q. Kang, E. Lim, Y. Jun, X. Jin, F. P. Payne, S. Alam, and D. J. Richardson, "Gain equalization of a six-modegroup ring core multimode EDFA," in The 40th European Conference and Exhibition on Optical Communication (ECOC, 2014), paper P.1.14.

[23] Q. Kang, P. Gregg, Y. Jung, E. L. Lim, S. Alam, S. Ramachandran, and D. J. Richardson, "Amplification of 12 OAM Modes in an air-core erbium doped fiber," Opt. Express 23, 28341-28348 (2015).

[24] Y. Jung, Q. Kang, S. Yoo, S. Raghuraman, D. Ho, P. Gregg, S. Ramachandran, S. Alam, and D. J. Richardson, "Optical Orbital Angular Momentum Amplifier based on an Air-Core Erbium Doped Fiber," in Optical Fiber Communication Conference Postdeadline Papers, OSA Technical Digest (online) (Optical Society of America, 2016), paper Th5A.5.

Yongmin Jung (SM'16) received the B.S. degree in Physics in 2003 from Chum-Ang University, Korea and the M.S. and Ph. $\mathrm{D}$ degree in engineering the information and communications from Gwangju Institute of Science and Technology (GIST), Korea, in 2004 and 2008, respectively. He joined Optoelectronics Research Centre (ORC), University of Southampton in U.K. in 2008 and is currently a senior research fellow. His main research interests are the space division multiplexing (SDM) and their optical amplifiers.

Qiongyue Kang was born in Hunan, China, in 1990. She received a $1^{\text {st }}$ class BEng degree in Electronics Engineering from University of Birmingham and Huazhong University of
Science and Technology, in 2011. She completed her PhD in photonics from the Optoelectronics Research Centre (ORC), University of Southampton in 2015. She is currently a postdoctoral research fellow at the ORC.

Hongyan Zhou, received her M.S degree in optical fiber communication from Beijing University of Posts and Telecommunications, China. She is currently an engineer of R\&D center of Yangtze Optical Fibre and Cable Co. Ltd. Now she works in the new fiber product R\&D and fiber test and transmission system test field.

\section{Rui Zhang, biography}

\section{Su Chen, biography}

Honghai Wang, received the Ph.D degree in opto-electronics from Huazhong University of Science and Technology, China in 1998. He is a senior expect in Yangtze Optical Fibre and Cable Co. Ltd., and now is the manager of R\&D department of YOFC. His research interests includes polarization maintaining optical fiber and many kinds of special optical fibers.

Yucheng Yang, received the Ph.D degree in condensed matter physics from Wuhan University, China in 2014. He is now in charge of the active optical fiber research in Yangtze Optical Fibre and Cable Co. Ltd. His research interests includes Rare earth doped optical fiber design and preparation technology.

Xianqing Jin received the Ph.D. degree in optical communications from Bangor University, Bangor, Gwynedd, U.K., in 2010. He is currently a research professor at the University of Science and Technology of China, Hefei, China. During the Ph.D. degree, he focused on the field of optical OFDM for next generation local area networks and access networks, including the real-time DSP design/implementation for high-speed optical OFDM transmission. Since 2012 to 2015, he has been a Postdoctoral Researcher at the University of Oxford, Oxford, U.K., he also studied the spatial multiplexing over ring-core fibres for future ultrahigh-speed optical networks. His research interests include topics in optical OFDM, spatial multiplexing, and real-time DSP for future optical communications.

Frank P. Payne is currently a Reader Emeritus in electronic materials at Oxford University, Oxford, U.K., and a Fellow of Lincoln College, Oxford. He has held senior appointments in the design of optical communication systems at Bookham Technology (now Oclaro), San Jose, CA, USA, and Nortel Networks, Mississauga, ON, Canada, working especially on 40-Gb/s WDM systems. He has also held appointments at Cambridge, Southampton, and Liverpool Universities. His research interests include the quantum field theory of optical devices and all areas of optical communications, especially the theory of optical waveguide components and systems.

Shaif-Ul Alam received his B.Sc. and M.Sc. degrees in physics from the University of Dhaka, Bangladesh, in 1988 and 1989 respectively. He is currently a Principal Research Fellow at the 
Optoelectronics Research Centre, University of Southampton, UK from where he received his Ph.D. degree in 2000. Between 2000 and 2008 he worked for SPI Lasers (UK) Limited and Quantronix Corporation in the USA and successfully led numerous high power fiber laser product developments. He is a recipient of prestigious Commonwealth Scholarship and JSPS Fellowship. He is a senior member of the OSA. His current research interests include fiber amplifier technology and applications of high power, short pulse fibre lasers. He has published over 200 articles in international scientific journals and conferences.

David J. Richardson (F'14) received B.Sc. and Ph.D. degrees in fundamental physics from Sussex University, Brighton, U.K. in 1985 and 1989 respectively. He joined the Optoelectronics Research Centre (ORC) at Southampton University in 1989 and was awarded a Royal Society University Fellowship in 1991 in recognition of his pioneering work on short pulsed fiber lasers. $\mathrm{He}$ is currently a Deputy Director of the ORC with responsibility for the ORC's fiber related activities. His current research interests include amongst others: optical fiber communications, microstructured optical fibers and pulsed high-power fiber lasers. $\mathrm{He}$ is a prominent figure in the international photonics community and has published more than 1200 conference and journal papers and produced more than 25 patents. As well as being a Fellow of the Institute of Electronic and Electrical Engineering, Prof. Richardson is a Fellow of the Optical Society of America, the Institute of Engineering and Technology. He became a Fellow of the Royal Academy of Engineering in 2009. 